\title{
P02.176. Acupuncture for symptom management in hemodialysis patients: a prospective, observational pilot study
}

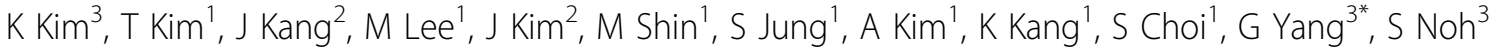 \\ From International Research Congress on Integrative Medicine and Health 2012 \\ Portland, Oregon, USA. 15-18 May 2012
}

\section{Purpose}

Patients undergoing hemodialysis suffer from a variety of complications related to end-stage renal disease. This prospective, observational pilot study aims to determine the feasibility, safety, and possible benefits of acupuncture for symptom management in patients undergoing hemodialysis.

\section{Methods}

Twenty-four patients undergoing hemodialysis received acupuncture treatment for their symptoms. Manually stimulated, individualized acupuncture treatments were provided twice a week for 6 consecutive weeks on a nondialysis day or on the day of hemodialysis prior to initiating treatment. Symptoms were evaluated using the Measure Your Medical Outcome Profiles 2 Questionnaire, and quality of life was measured by the Kidney Disease Quality of Life-Short Form (KDQOL-SFTM) Version 1.3 at baseline, 7 weeks and 11 weeks from baseline. Statistical analysis was conducted on the basis of the intention-totreat principle.

\section{Results}

Twenty-one patients (87\%) completed the whole treatment course and follow-up evaluation. Three patients dropped out due to increased fatigue $(n=1)$, pancreatic and renal transplantation $(\mathrm{n}=1)$, and infections of the ateriovenous fistula used for hemodialysis access $(n=1)$. Patients experienced a significant improvement of symptoms considered the most bothersome, reporting a decrease of 1.87 and 2.08 points on a 0-6 symptom scale at 7 weeks and 11 weeks, respectively (both $\mathrm{p}<0.0001$ ).
Some subscales of KDQOL-SF( $\left.{ }^{\mathrm{TM}}\right)$ showed significant improvement at 7 weeks (effects of kidney disease, burden of kidney disease, role-limitations physical, emotional well-being, and energy/fatigue) and 11 weeks (physical functioning and energy/fatigue). No serious adverse events related to acupuncture occurred.

\section{Conclusion}

Acupuncture seems feasible and safe for symptom management in patients undergoing hemodialysis. Future controlled trials are needed to confirm the benefits of acupuncture.

\section{Author details}

${ }^{1}$ Korea Institute of Oriental Medicine, Daejeon, Republic of Korea. ${ }^{2}$ Kyung Hee University, Seoul, Republic of Korea. ${ }^{3}$ School of Korean Medicine, Pusan National University, Yangsan, Republic of Korea.

Published: 12 June 2012

doi:10.1186/1472-6882-12-S1-P232

Cite this article as: Kim et al:: P02.176. Acupuncture for symptom management in hemodialysis patients: a prospective, observational pilot study. BMC Complementary and Alternative Medicine 2012 12(Suppl 1): P232.

\footnotetext{
${ }^{3}$ School of Korean Medicine, Pusan National University, Yangsan, Republic of Korea

Full list of author information is available at the end of the article
} 